\title{
Habilidades básicas para aprender a pensar
}

\author{
Marta Izaguirre Quezada*
}

mizaguirre@lasallep.edu.mx

María Celina Arredondo

Trillas, 2006

Este libro plantea de manera sencilla un tema muy complejo como es el de las habilidades de pensamiento. La obra está integrada por cuatro capítulos teóricos y dos prácticos los cuales ofrecen una serie de ejercicios para practicar las habilidades básicas del pensamiento y ejercicios de metacognición.

En el primer capítulo se aborda la concepción de la inteligencia. La autora hace un recuento de lo que diversos investigadores han planteado de este concepto. Identifica como características en común de esta noción: la capacidad para adaptarse al medio; la abstracción y la conciencia (cfr. p. 18). La autora concibe a la inteligencia y al aprendizaje como un proceso constructivo e interactivo.

En el segundo capítulo aborda el concepto de pensamiento. Hace un breve reconocimiento de los estadíos del desarrollo cognitivo que establece Piaget. Plantea las cualidades del pensamiento, como la autocrítica, que permite ver los errores y descubrir aciertos. La flexibilidad del pensamiento que considera como la posibilidad de encontrar nuevas soluciones a una problemática. Otra cualidad fundamental que se resalta del pensamiento es el orden lógico y metodológico que se utiliza al examinar problemáticas.

Finalmente el capítulo dos aborda el concepto de habilidades del pensamiento. Hace un recuento de las distintas concepciones y clasificaciones desde diferentes perspectivas de lo que son habilidades para llegar a explicar lo que son las habilidades cognitivas.

De manera esquemática y sencilla presenta las habilidades básicas del pensamiento como son comparar, relacionar, hacer analogías, clasificar, discriminar, asociar, entre otras (cfr. p. 28). Una idea fundamental que sostiene es que es posible enseñar estas habilidades de pensamiento, no como algo opuesto a enseñar el contenido propio de cada asignatura, sino de manera complementaria. 
Estas habilidades son la base para alcanzar procesos de pensamiento más complejos. La autora propone ejercitar estas habilidades en un programa paralelo a los contenidos propios de la asignatura, enseñado por el mismo docente. El dominio de estas habilidades básicas conforman las capacidades del individuo que redunda en su razonamiento y nivel de inteligencia,

siempre en interacción con el pensamiento. Esto lo presenta de manera gráfica en una figura piramidal en donde en la base están las habilidades, en un segundo nivel las capacidades, le sigue el razonamiento, y en la cúspide la inteligencia; todos los niveles en interacción con el pensamiento. (cfr. p. 31). Hace referencia a las pruebas cognitivas que existen enumerando de manera esquemática los factores de aptitud que evalúan.

En el tercer capítulo, de manera muy sucinta retoma los elementos y factores que intervienen en el proceso didáctico. Destaca que la enseñanza debe ir más allá de la transmisión de conocimientos y hace énfasis en el desarrollo de habilidades. Al alumno lo concibe como un procesador activo que posee habilidades cognitivas para aprender y solucionar problemas que lo llevarán a desarrollar diferentes competencias cognitivas. Al profesor lo sitúa como un mediador o facilitador que no sólo debe transmitir conocimientos sino que debe enseñar a pensar a sus alumnos. En cuanto al programa destaca en su propuesta que además de cubrir los contenidos curriculares es importante incluir los ejercicios que la autora propone (u otros similares) en los capítulos posteriores. Puntualiza recomendaciones precisas al tratar los objetivos, la realización y evaluación de procesos enseñanza-aprendizaje; hace recomendaciones muy puntuales en cuanto a la motivación, las actitudes, el tiempo, la transferencia de aprendizaje y el entorno que favorece dichos procesos.

El capítulo cuatro presenta una serie de ejercicios para desarrollar las diferentes habilidades básicas que propone en el capítulo dos.

El capítulo cinco define de manera muy accesible a la cognición como a la acción y efecto de conocer. Retoma a Kagan y Lang (1978, en Habilidades básicas para aprender a pensar p. 73) para hablar de cognición de manera global como los procesos que una persona involucra en: "la extracción de información del mundo exterior; la aplicación de conocimiento previo a la información recientemente percibida; la integración de ambas para crear nuevos conocimientos; el almacenaje de la información en la memoria para poder recuperarla y usarla después; la evaluación continua de la calidad y coherencia lógica de los procesos y productos mentales de la persona“. Define a la metacognición como la "conciencia que se tiene de los procesos intelectuales empleados para realizar determinada tarea o alcanzar una meta" (p. 74). Después de analizar las 
concepciones de diferentes autores infiere que los procesos básicos de la metacognición son la planificación, control y evaluación que se traducen en "tres actividades básicas: planificación, ¿qué estrategias?; ejecución: ¿cómo se aplican?; evaluación: ¿qué procesos sirvieron para llegar a la meta?” (p.75)

La utilidad de la metacognición es permitir identificar los procesos intelectuales correctos para cada tarea (cfr. p. 77). El profesor debe ser consciente de sus propios procesos metacognitivos y compartirlos con los alumnos. Como aplicaciones en el aprendizaje de este proceso ejemplifica la comprensión lectora diferenciando seis modos distintos de lectura y cómo es que aplicando la metacognición puede mejorar esta comprensión. Por último, plantea cómo la metamemoria, "conocimiento y control de los procesos de memoria, su capacidad, sus limitaciones, su operatividad" (p. 81) hacen más eficiente la lectura. El capítulo plantea ejercicios básicos de metacognición dividiéndolos en metacomprensión, solución de problemas, comprensión de lectura, y metamemoria.

El acierto de este libro, es presentar de manera accesible y didáctica para los docentes una temática que en sí es compleja. Su propuesta de que cada docente elabore ejercicios similares o retome los que se proponen para "enseñar a pensar a los alumnos" además del programa propio de la asignatura, no me parece tan sencilla de llevarla a la práctica. Sin embargo, es cierto, que como docente hay ocasiones en que no puedes ayudar a aprender a los alumnos, dadas las limitaciones de éstos en cuanto a habilidades básicas, por lo que hay que incidir sobre ellas para ayudar en el proceso de construcción del alumno. Es una propuesta retadora que habría que experimentar. 